Computer Optics and Nanophotonics

\title{
SIMULATION OF OPTICAL SIGNALS PROPAGATION IN A RANDOM MEDIA
}

\author{
M.S. Kirilenko, S.N. Khonina \\ Samara National Research University, Samara, Russia, \\ Image Processing Systems Institute - Branch of the Federal Scientific Research Centre "Crys- \\ tallography and Photonics" of Russian Academy of Sciences, Samara, Russia
}

\begin{abstract}
The operator of the optical beam propagation through turbulent environment using the Fresnel approximation is being considered. The correlation function of random field describing inhomogeneous medium is given in the form of Gaussian function. The process of random field modeling using the Fourier transform is demonstrated. A selective correlation function is calculated, the deviation from the preset one is defined. The intensity distributions after propagation of optical beams in free space and in a random medium are given. As the input beam such optical distributions as Hermite - Gauss modes, rectangular pulse, and vortex beams were considered.
\end{abstract}

Keywords: turbulent media, random field, Fourier transform, Fresnel transform, Gaussian modes, vortex beams.

Citation: Kirilenko MS, Khonina SN. Simulation of optical signals propagation in a random media. CEUR Workshop Proceedings, 2016; 1638: 55-65. DOI: 10.18287/1613-0073-2016-1638-55-65

\section{Introduction}

Distribution of the optical signal in a free space can be subject to distortions associated with the turbulence of the medium [1,2]. Due to the properties of turbulence random changes in atmospheric refractive index can cause distortion of the laser radiation intensity. Classical methods for describing wave propagation through the turbulent atmosphere are based on the Rytov method applications [3] and the method of parabolic equations [4], connection between them has been demonstrated previously [5].

With the help of these methods propagation of optical signals from partially coherent source has been investigated [6,7], and also features of propagation of different laser beams in a turbulent medium have been studied, including Gaussian beams of higher orders [8], hollow beams [9], diffraction-free beams, such as Gauss-Bessel and Eerie beams $[10,11]$, as well as cosine beams [12]. Wherein it was found that the higherorder Gaussian beams, including vortex bundles [13], as well as various spatially structured bundles become broader under the influence of turbulence in a lesser de- 
gree than the fundamental Gaussian beam. In addition, the spread of beams with an inhomogeneous polarization in a turbulent medium was studied $[14,15]$.

The most convenient and effective means of forming random beams with specified properties are the methods of diffraction optics [16-20].

In this paper modeling of random optical beams through random medium with a given correlation function in the form of the Gaussian function is being considered. The comparative calculations of the propagation of Gaussian modes (Hermite-Gaussian and Laguerre-Gaussian) through the random medium are being performed.

\section{Passing through the random medium}

The distribution of a laser beam in a random medium can be described on the basis of the integral expressing the extended Huygens-Fresnel principle [8, 21]:

$$
\begin{aligned}
& E(u, v, z, t)=-\frac{i k}{2 \pi z} \exp (i k z) \times \\
& \times \int_{-\infty}^{\infty} \int_{-\infty}^{\infty} E_{0}(x, y) \exp \left\{\frac{i k}{2 z}\left[(x-u)^{2}+(y-v)^{2}\right]+\Psi(x, y, u, v, z)-i \omega t\right\} \mathrm{d} x \mathrm{~d} y,
\end{aligned}
$$

where $E_{0}(x, y)$ is a field in the input plane (at $\left.z=0\right), E(u, v, z, t)$ is a field at a distance $z$ from the input plane, $\Psi(x, y, u, v, z)$ is a random part in the propagation operator, related to atmospheric turbulence, $\omega$ is the frequency of laser oscillation, $t$ is time.

Equation (1) corresponds to the Rytov method [21], and the function $\Psi(x, y, u, v, z)$ describes accidental deviations of the phase function of a spherical wave, propagating from the initial to the output plane.

Note that in this way the complex phase can be implemented also in other integral transforms, for example, into fractional Fourier transform [22, 23] which describes propagation of optical signal in parabolic fiber [24, 25].

Consider the one-dimensional case for simplicity. Let the correlation function of the random field has the shape of a Gaussian function:

$$
\begin{aligned}
& R\left\{\exp \left[\psi\left(x_{1}, u_{1}, 0\right)\right], \exp \left[\psi\left(x_{2}, u_{2}, z\right)\right]\right\}= \\
& =A \exp \left[-\frac{1}{\sigma_{x}^{2}}\left(x_{1}-x_{2}\right)^{2}\right]=R_{\exp [\psi]}\left(\left|x_{1}-x_{2}\right|, z\right),
\end{aligned}
$$

where $A>0$ and $\sigma_{x}>0$. Note that this correlation function depends on the module of difference between the coordinates $x_{1}$ and $x_{2}$, but not on each one of them individually. Moreover, it does not depend on the coordinates in the output plane $u_{1}$ and $u_{2}$, but it depends only on the distance $z$ to it. 
Let us assume that the average intensity of the beam passing through a turbulent atmosphere is maintained, and for this we'll define the average amplitude of the random field equal to one:

$$
\langle\exp [\psi(x, u, z)]\rangle=1
$$

\section{Generation of random field}

Without loss of generality let us consider a random field with zero mathematical expectation, ie, a field of the type:

$U(x, u, z)=\exp [\psi(x, u, z)]-\langle\exp [\psi(x, u, z)]\rangle$,

since after its modeling it is easy to get the desired selection:

$\exp [\psi(x, u, z)]=U(x, u, z)+\langle\exp [\psi(x, u, z)]\rangle$.

Wherein the correlation function remains unchanged:

$$
R_{\exp [\psi]}\left(x_{1}-x_{2}, z\right)=R_{U}\left(x_{1}-x_{2}, z\right)=R_{U}(x, z) \text {. }
$$

Let us drop all the variables, except $x$, and assume that the required random field $U(x)$ can be obtained through the passage of a complex-valued white noise $\xi(x)$ with a unit dispersion and a correlation function equal to the Dirac delta function $\delta(x)$, through a linear filter:

$U(x)=h(x) * \xi(x)$,

where the asterisk * denotes convolution operation, $h(x)$ is a determined function (pulse characteristic). Then the generation problem reduces to finding $h(x)$ function.

By definition, the correlation function $U(x)$ is equal to: $R_{U}(x)=\left\langle U\left(x+x^{\prime}\right) U^{*}\left(x^{\prime}\right)\right\rangle=$

$=\left\langle\int_{-\infty}^{\infty} h(\alpha) \xi\left(x+x^{\prime}-\alpha\right) d \alpha \int_{-\infty}^{\infty} h^{*}(\beta) \xi^{*}\left(x^{\prime}-\beta\right) d \beta\right\rangle=$

$=\iint_{2} h(\alpha) h^{*}(\beta)\left\langle\xi\left(x+x^{\prime}-\alpha\right) \xi^{*}\left(x^{\prime}-\beta\right)\right\rangle d \alpha d \beta=$

$=\iint_{\Omega^{2}} h(\alpha) h^{*}(\beta) R_{\xi}(x-\alpha+\beta) d \alpha d \beta=$

$=\iint_{2} h(\alpha) h^{*}(\beta) \delta(x-\alpha+\beta) d \alpha d \beta=$

$=\int_{-\infty}^{\infty} h(x+\beta) h^{*}(\beta) d \beta$. 
Thus, we find that the correlation function $R_{U}(x)$ can be defined as autocorrelation of the function $h(x)$ :

$R_{U}(x)=h(x) \otimes h(x)$,

where the $\otimes$ symbol denotes the operation of mutual correlation. Using the Fourier transformation $\mathfrak{J}$, we get:

$$
\Im\left[R_{U}(x)\right]=|\Im[h(x)]|^{2},
$$

from where

$$
\Im[h(x)]=\sqrt{\Im\left[R_{U}(x)\right]} .
$$

The last formula makes it possible to find the $h(x)$ function, using the inverse Fourier transform. However, for calculation according to formula (7) the easiest way is to reuse the Fourier transform to avoid fold operation:

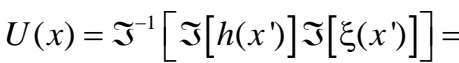

$$
\begin{aligned}
& =\mathfrak{J}^{-1}\left[\sqrt{\mathfrak{J}\left[R_{U}\left(x^{\prime}\right)\right]} \mathfrak{\Im}\left[\xi\left(x^{\prime}\right)\right]\right] \text {. }
\end{aligned}
$$

Modeling of $U(x)$ random field can be realized by the formula (12) using a fast Fourier transform algorithm. Note that the Fourier transform from white noise will also be white noise.

\section{$4 \quad$ Modeling of the one-dimensional signals propagation}

In the modeling process the following parameters were used: the wavelength $\lambda=2 \pi / k=633 \mathrm{~nm}$, the input region width $[-a ; a]=[-60 \mathrm{~mm} ; 60 \mathrm{~mm}], A=1$, $\sigma_{x}=1.5 \mathrm{~m}$. Fresnel transformation formulas (1) and random field generations (12) were implemented using fast Fourier transform.

Input optical distribution passes consecutively 6 times through the Fresnel transformation (1) with $z=10 \mathrm{~m}$; thus, the field extends over 60 meters.

Note that the passage of 60 meters using only one transformation does not change the overall picture, as the random field has a correlation function that depends on the propagation distance.

Figures 1a, $1 \mathrm{~b}$ are examples of generating a random field in the region $[-250 \mathrm{~mm} ; 250 \mathrm{~mm}]$.

The view of the correlation function and of the selective correlation function is shown in Figure 2. 

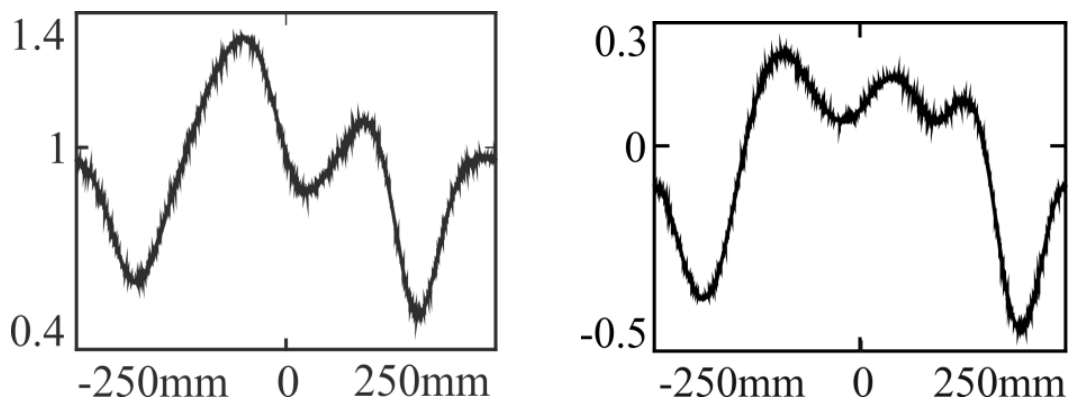

Fig. 1. Example of a random field: amplitude and phase

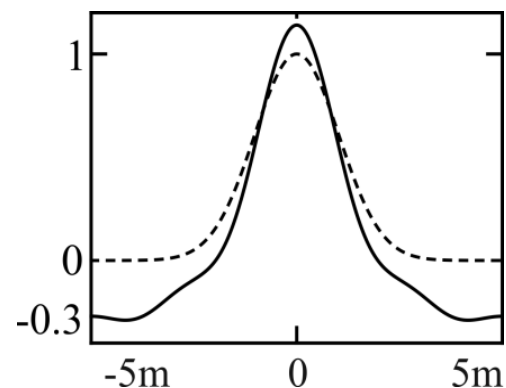

Fig. 2. Sample correlation function (solid line) compared with a given (dotted line)

As the input beam Gaussian function $f_{1}(x)$ ((Hermite-Gaussian mode of zero order), Hermite-Gaussian mode of the fourth order $f_{2}(x)$, and rectangular function $f_{3}(x)$ :

$$
\begin{aligned}
& f_{1}(x)=\exp \left(-x^{2} / 2 \sigma_{f}^{2}\right), \\
& f_{2}(x)=\exp \left(-x^{2} / 2 \sigma_{f}^{2}\right) H_{4}\left(x / \sigma_{f}\right), \\
& f_{3}(x)=\operatorname{rect}(x / 2 \gamma)= \begin{cases}1, & |x| \leq \gamma, \\
0, & |x|>\gamma,\end{cases}
\end{aligned}
$$

where $\sigma_{f}=1 \mathrm{~mm}, \gamma=30 \mathrm{~mm}, H_{4}\left(x / \sigma_{f}\right)$ is the fourth Hermite polynomial.

The results are shown in Figures 3, 4, and 5, respectively, in comparison with the propagation of the field in free space without irregularities.

\section{$5 \quad$ Modeling of the singular Gaussian beams propagation}

In analogy to the one-dimensional case we can consider the distribution of optical beams in two-dimensions variant. The difference will consist only in the fact that the corresponding one-dimensional transformations are replaced by the two-dimensional ones. 

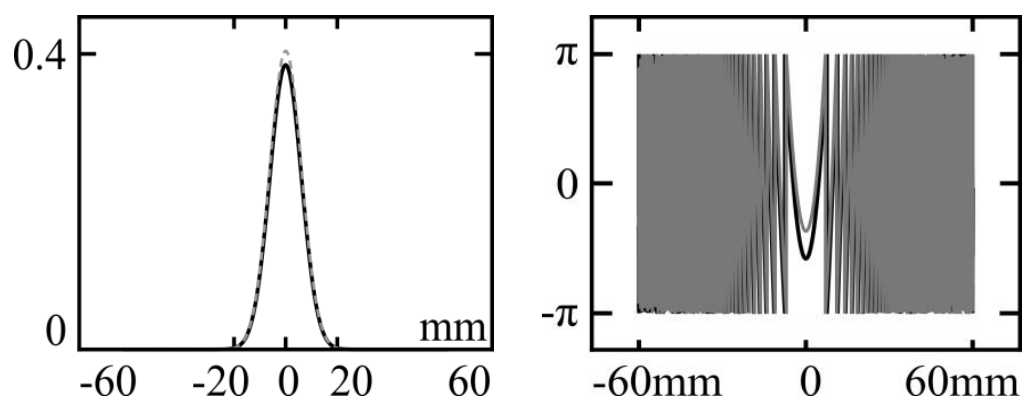

Fig. 3. Propagation of a Gaussian beam in free space (gray graph) and in a turbulent medium (black graph): the amplitude and phase
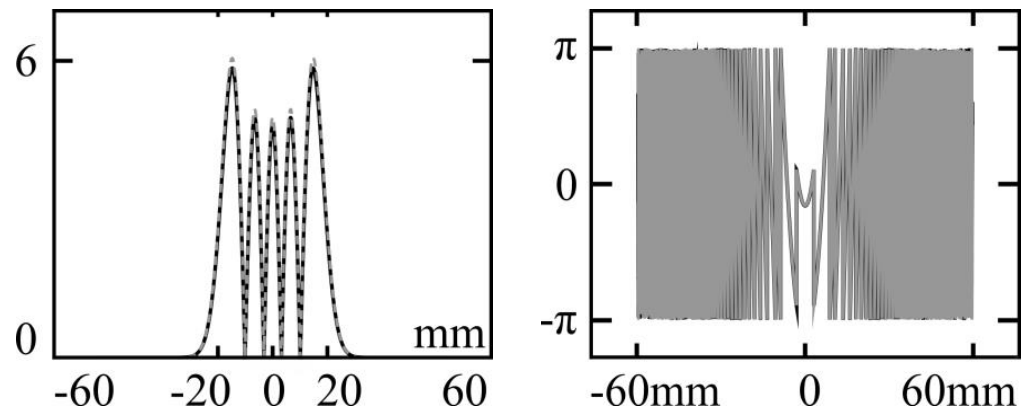

Fig. 4. Propagation of Hermite-Gauss mode in free space (gray graph) and in a turbulent medium (black graph): the amplitude and phase
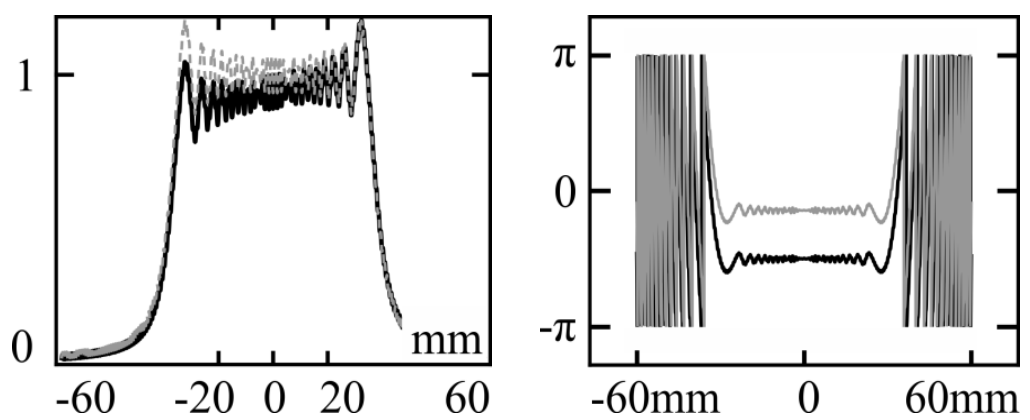

Fig. 5. Rectangular signal propagation in free space (gray graph) and in a turbulent medium (black graph): the amplitude and phase

Figure 6 shows an example of generating a random two-dimensional field with correlation function in the form of Gaussian function. Figure 7 shows an image of a sample correlation function. 

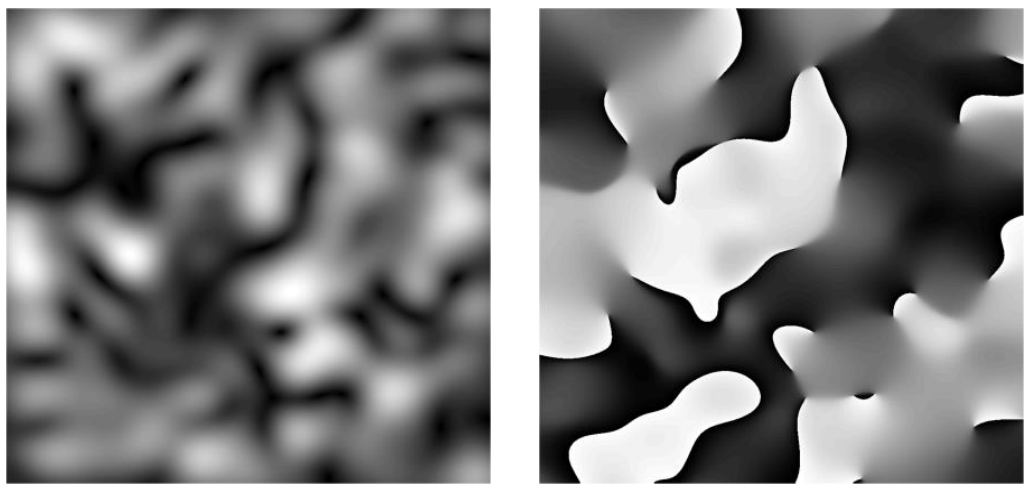

Fig. 6. Generation of a random two-dimensional field: amplitude and phase

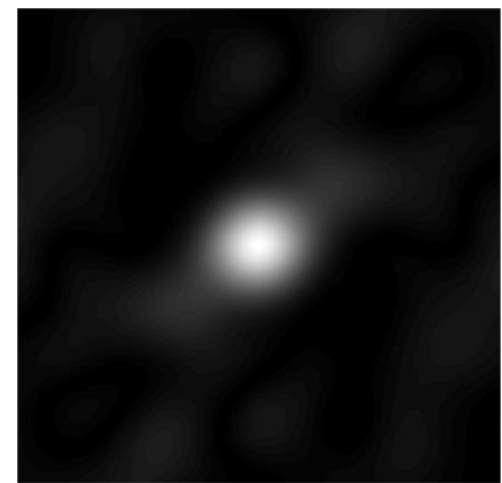

Fig. 7. Sample correlation function of the two-dimensional field

Hermite-Gauss mode is selected as an example of the optical beam propagation through random field [26-28]. The result is shown in Figure 8.
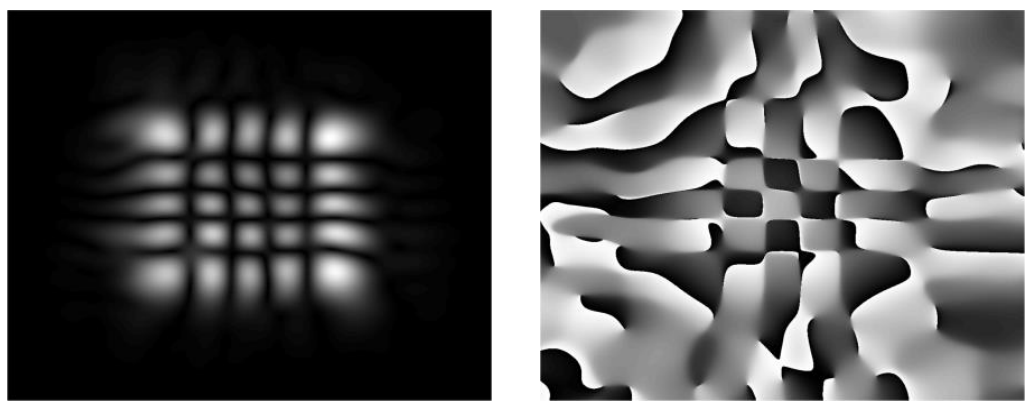

Fig. 8. Hermite-Gauss mode after passing through the random field: amplitude and phase

In addition to the extended Huygens-Fresnel principle (1), there is a different approach for modeling random medium, based on the scheme with screens with a random phase $[29,30]$. 
Table 1 shows the results of comparative modeling of propagation of the fundamental Gaussian beam and of vortex Laguerre-Gaussian laser beams [26, 31, 32] in a random medium. A lot of thin phase screens with a random distribution, separated by a free space, were used for modeling. Thus, the beam periodically acquires random phase changes in the thin screen, passes part of the way in free space. During the modeling the screens with uniform random phase noise in the range of $[0, \pi]$ were located every $1.5 \mathrm{~km}$ of the passage way of $15 \mathrm{~km}$.

As follows from the given results of the modeling, at a distance of $15 \mathrm{~km}$ the sizes of the Gaussian and of the vortex beams become virtually identical, although originally the Gaussian beam was more compact. Note that the vortex phase structure of the Laguerre-Gaussian beam remains sufficiently expressed despite the significant noise and the distance covered.

The stability of the vortex beam to turbulent impact was noted in the work [13], where it was stated that the vortex beam can "split, deviate, wander" outside the area of the detector, but will never disappear. In this work it was shown that a vortex beam of the fifth order is stored in the turbulent medium for over 2 kilometers, and is then splits into first order vortices that are lasting longer than 10 kilometers.

\section{Conclusion}

The operator, based on extended Huygens-Fresnel principle, of optical beam propagation through turbulence media was considered. The generation of a random field describing the inhomogeneous media with a given correlation function is implemented by linear filtering of white noise in the spectral domain. Deviation between the calculated correlation function and the given correlation function showed good performance of this algorithm. The computations of intensity distributions of Gaussian beams of high order were performed in the free space and random media.

Less broadening of the vortex beam in comparison with fundamental beam was shown based on comparative modeling of fundamental Gaussian beam and vortex laser beam propagation in random media.

\section{Acknowledgement}

This work was financially supported by the Russian Science Foundation (grant 14-3100014). 
Table 1. Results of modeling of laser beam propagation with periodical phase noising of complex beam distribution

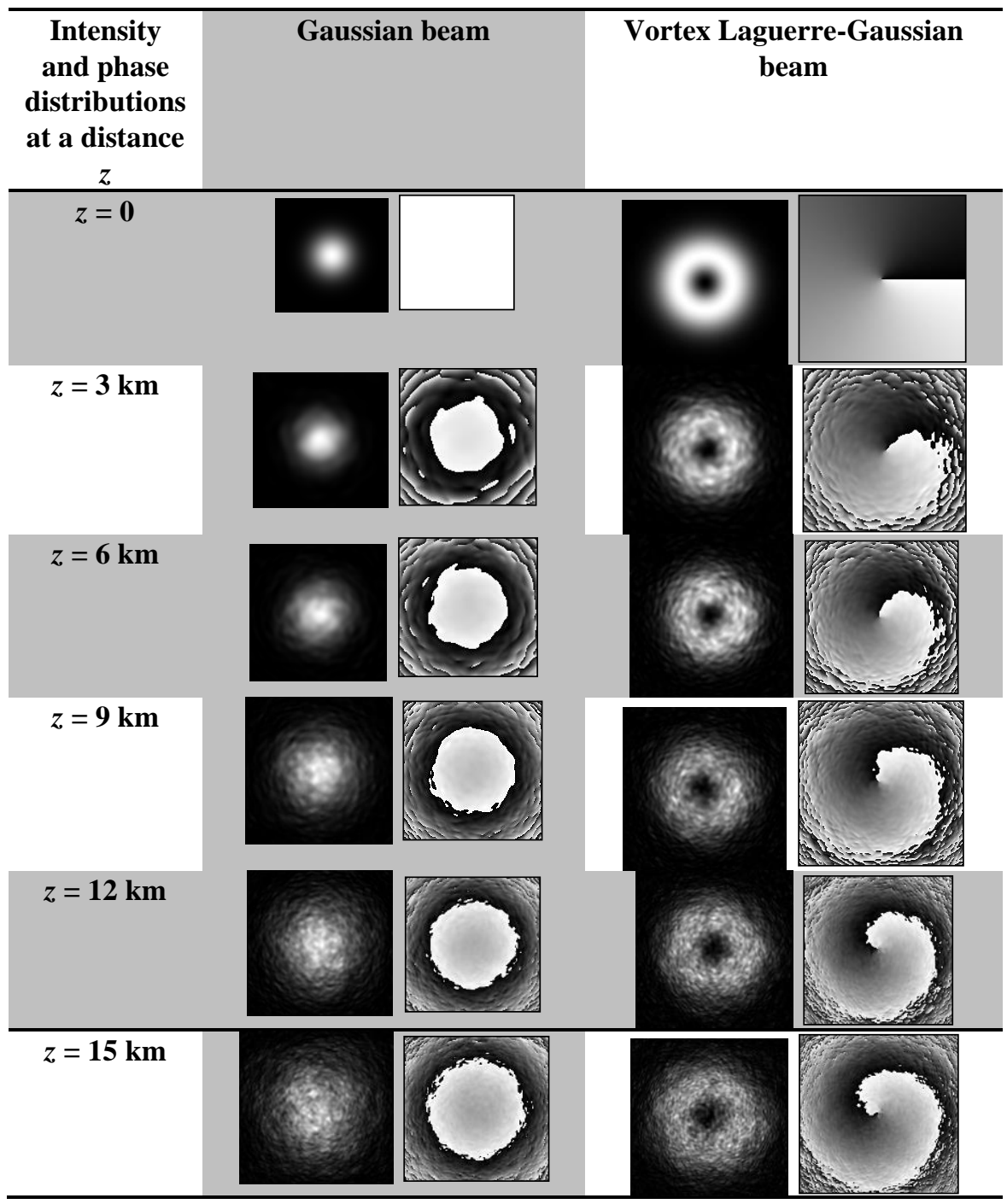

\section{References}

1. Grabner M, Kvicera V. Measurement of the structure constant of refractivity at optical wavelengths using a Scintillometer. Radioengineering, 2012; 21(1): 455-458.

2. Majumdar AK, Ricklin JC. Free-space laser communications: principles and advances, Vol. 2. New York: Springer Science \& Business Media, 2008: 418 p. 
3. Tatarskii VI. Wave Propagation in a Turbulent Medium. New York: McGraw-Hill, 1961: $285 \mathrm{p}$.

4. Tatarskii VI. The Effects of the Turbulent Atmosphere on Wave Propagation. Jerusalem: Israel Program for Scientific Translations, 1971: $472 \mathrm{p}$.

5. Manning RM. The relationship between the solutions of the parabolic equation method and first Rytov approximation in stochastic wave propagation theory. Waves in Random and Complex Media, 2008; 18(4): 615-621.

6. Ricklin JC, Davidson FM. Atmospheric turbulence effects on a partially coherent Gaussian beam: implications for free-space laser communication. Journal of the Optical Society of America A, 2002; 19(9): 1794-1802.

7. Wang SCH, Plonus MA. Optical beam propagation for a partially coherent source in the turbulent atmosphere. Journal of the Optical Society of America A, 1979; 69(9): 12971304.

8. Young CY, Gilchrest YV, Macon BR. Turbulence induced beam spreading of higher order mode optical waves. Opt. Eng., 2002; 41(5): 1097-1103.

9. Cai Y, He S. Propagation of various dark hollow beams in a turbulent atmosphere. Opt. Express, 2006; 14(4): 1353-1367.

10. Eyyuboglu HT. Propagation of higher order Bessel-Gaussian beams in turbulence. Appl. Phys. B, 2007; 88(2): 259-265.

11. Chu X. Evolution of an Airy beam in turbulence. Opt. Lett., 2011; 36(14): 2701-2703.

12. Zhou G. Propagation of a higher-order cosh-Gaussian beam in turbulent atmosphere. Optics express, 2011; 19(5): 3945-3951.

13. Gbur G, Tyson RK. Vortex beam propagation through atmospheric turbulence and topological charge conservation. J. Opt. Soc. Am. A, 2008; 25(1): 225-230.

14. Gu Y, Korotkova O, Gbur G. Scintillation of nonuniformly polarized beams in atmospheric turbulence. Opt. Lett., 2009; 34(15): 2261-2263.

15. Jacks HC, Korotkova O. Polarization and intensity correlations in stochastic electromagnetic beams upon interaction with devices of polarization optics. Appl Phys B, 2011; 103: 413-419.

16. Golovashkin DL, Kotlyar VV, Soifer VA, Doskolovich LL, Kazanskiy NL, Pavelyev VS, Khonina SN, Skidanov RV. Computer Design of Diffractive Optics. Edited by V.A. Soifer. Cambridge Inter. Scien. Pub. Ltd. \& Woodhead Pub. Ltd., 2012: 896 p.

17. Khonina SN, Kotlyar VV, Soifer VA, Honkanen M, Lautanen J, Turunen J. Generation of rotating Gauss-Laguerre modes with binary-phase diffractive optics. Journal of Modern Optics, 1999; 46(2): 227-238.

18. Kotlyar VV, Kovalev AA, Skidanov RV, Khonina SN, Turunen J. Generating hypergeometric laser beams with a diffractive optical element. Applied Optics, 2008; 47(32): 61246133.

19. Khonina SN, Balalayev SA, Skidanov RV, Kotlyar VV, Paivanranta B, Turunen J. Encoded binary diffractive element to form hyper-geometric laser beams. Journal of Optics A: Pure and Applied Optics, 2009; 11: 065702-7pp.

20. Khonina SN. Specular and vortical Airy beams. Optics Communications, 2011; 284: 4263-4271.

21. Feizulin ZI, Kravtsov YA. Broadening of a laser beam in a turbulent medium. Radiophys. Quantum Electron, 1967; 10 (1): 33-35.

22. Namias V. The fractional Fourier transform and its application in quantum mechanics. Journal of the Institute of Mathematics and its Applications, 1980; 25: 241-265.

23. Alieva T, Bastiaans MJ, Calvo ML. Fractional transforms in optical information processing. EURASIP Journal on Applied Signal Processing, 2005; 10: 1-22. 
24. Khonina SN, Striletz AS, Kovalev AA, Kotlyar VV. Propagation of laser vortex beams in a parabolic optical fiber. Proceedings SPIE, 2010; 7523: 75230B-1-12.

25. Kirilenko MS, Zubtsov RO, Khonina SN. Calculation of eigenfunctions of a bounded fractional Fourier transform. Computer Optics, 2015; 39(3): 332-338. [in Russian] DOI: 10.18287/0134-2452-2015-39-3-332-338.

26. Yariv A. Introduction to Optical Electronics. Moscow: "Vysshaya shkola" Publisher, 1983: 400 p. [in Russian]

27. Kotlyar VV, Khonina SN, Soifer VA. Generalized Hermite beams in free space. Optik, 1998; 108(1): 20-26.

28. Khonina SN, Kotlyar VV, Soifer VA. Self-reproduction of multimode Hermite-Gaussian beams. Technical Physics Letters, 1999; 25(6): 489-491.

29. Tian Y, Guo J, Wang R, Wang T. Mathematic model analysis of Gaussian beam propagation through an arbitrary thickness random phase screen. Opt. Express, 2011; 19(19): 18216-18228.

30. Liu X, Pu J. Investigation on the scintillation reduction of elliptical vortex beams propagating in atmospheric turbulence. Optics Express, 2011; 19(27): 26444-26450.

31. Khonina SN, Kotlyar VV, Soifer VA. Diffraction optical elements matched to the GaussLaguerre modes. Optics and Spectroscopy, 1998; 85(4): 636-644.

32. Khonina SN, Kotlyar VV, Skidanov RV, Soifer VA, Laakkonen P, Turunen J. GaussLaguerre modes with different indices in prescribed diffraction orders of a diffractive phase element. Optics Communications, 2000; 175: 301-308. 